\title{
SIR-optimized Weighted Linear Parallel Interference Cancellers on Rayleigh Fading Channels
}

\author{
V. Tikiya, S. Manohar, and A. Chockalingam \\ Department of ECE, Indian Institute of Science, Bangalore 560012, India
}

\begin{abstract}
In this paper, we present weighted linear parallel interference cancellers (LPIC) where the estimated multiple access interference (MAI) from each interfering user is weighted by a factor before cancellation on Rayleigh fading and diversity channels. We obtain exact expressions for the signal-tointerference ratio (SIR) at the output of the cancellation stages which we maximize to obtain the optimum weights for each interfering user in each cancellation stage. For a system that uses same weight for all interfering users in a given stage, we obtain exact closed-form expressions for the optimum weights for the different stages of the LPIC. We show that this SIR-optimized weighted LPIC scheme clearly outperforms both the matched filter (MF) detector as well as the conventional LPIC (where the weight is taken to be unity for all interfering users and for all stages), in both near-far as well as non-near-far conditions.
\end{abstract}

Keywords - Linear parallel interference canceller, signal-to-interference ratio, fading channels.

\section{INTRODUCTION}

Parallel interference cancellation (PIC) is a multiuser detection technique which lends itself to a multistage implementation where the decision statistics of the users from the previous stage are used to estimate and cancel the MAI in the current stage, and a final decision statistic is obtained at the last stage [1]. When an estimate of the MAI is obtained from the hard bit decisions from the previous stage, it is termed as 'hard-decision PIC' (non-linear PIC) [2]. On the other hand, MAI estimates can be obtained using the soft values of the decision statistics from the previous stage, in which case the PIC is termed as linear PIC (LPIC) [3],[4]. LPICs have the advantages of implementation simplicity, analytical tractability, and good performance under certain conditions.

In a conventional LPIC, an estimate of the MAI for a desired user in a stage is obtained using all the other users' soft outputs from the previous stage. It is likely that these MAI estimates are inaccurate due to poor channel conditions (e.g., high interference, deep fades, etc.). Under such conditions, the cancellation can become ineffective to an extent that it may be better not to do cancellation. In fact, it has been known that the conventional LPIC performs worse than the MF detector (where no cancellation is done) at low SNRS, due to the poor accuracy of the MAI estimates at low SNRs. This can be alleviated by properly weighing the MAI estimates before cancellation [4]. A key question is how to choose the weights for different cancellation stages. An intuitive approach is to keep the value of the weight low at the early stages and large at the later stages, as done in [4], because the

This work was supported in part by the Swarnajayanti Fellowship, Department of Science and Technology, New Delhi, Government of India, under Project Ref: No.6/3/2002-S.F.
MAI estimates can be more reliable in the later stages since much of the MAI would have been cancelled by then. A more formal approach, which we adopt here, is to obtain appropriate functions (e.g., expressions for interference variance or SIR) which when optimized will give the optimum weights.

The issue of the choice of weights in LPIC has been addressed in [5]-[8], but only for AWGN channels. In this paper, we are concerned with SIR-optimized weighted LPICs for Rayleigh fading and diversity channels. Specifically, we derive exact expressions for the average SIR at the output of the cancellation stages, which we maximize to obtain the optimum weights for each interfering user in each cancellation stage. A key contribution in this paper is that, for a system that uses same weight for all interfering users in a given stage, we obtain exact closed-form expressions for the optimum weights for the different stages of the LPIC. We show that this SIR-optimized weighted LPIC clearly outperforms both the matched filter (MF) detector as well as the conventional LPIC (where the weight is taken to be unity for all interfering users and for all stages), in both near-far as well as non-near-far conditions.

\section{SYSTEM MODEL}

Consider a $K$-user synchronous CDMA system where the received signal is given by

$$
y(t)=\sum_{\mathrm{fc}=\mathrm{i}}^{K} A_{k} h_{k} b_{k} s_{k}(t)+n(t), \quad t \in[0, T],
$$

where $b_{k} €\{+1,-1\}$ is the bit transmitted by the fcth user, $T$ is one bit duration, $A_{k}$ is the transmit amplitude of the fcth user's signal, $h_{k}$ is the complex channel fade coefficient corresponding to the fcth user, $s_{k}(t)$ is the unit energy spreading waveform of the fcth user defined in the interval $[0, T]$, i.e., $10 s_{k}^{2}(t) d t=1$, and $n(t)$ is thewhite Gaussian noise with zero mean and variance $\sigma^{2}$. The fade coefficients $h k^{\prime} s$ are assumed to be i.i.d complex Gaussian r.v's (i.e., fade amplitudes are assumed to be Rayleigh distributed) with zero mean and $E\left[h_{I}^{2}\right]=E\left[h_{Q}^{2}\right]=1$, where $h_{k I}$ and $h_{k Q}$ are the real and imaginary parts of $h_{k}$. The channel fade is assumed to remain constant over one bit interval.

We consider a multistage LPIC at the receiver. The first stage is a conventional MF, which is a bank of $K$ correlators, each matched to a different user's spreading waveform. The received vector $\mathbf{y}^{(1)}$ at the output of the MF stage (the superscript (1) in $\mathbf{y}^{(1)}$ denotes the first stage) is given by

$$
\left.\mathbf{y}^{(1)}={ }_{\llcorner} y_{1}^{(1)}, y_{2}^{(1)}, \cdots, y_{K}^{(1)}\right],
$$


where $y_{k}^{\prime \prime}$ is the fcth user's MF output, given by

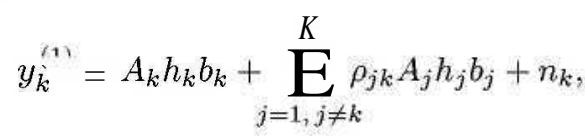

where $\rho_{j k}$ is the cross-correlation coefficient between $j$ th and fcth users' spreading waveforms, given by $p j k=\int_{0}^{1} s_{l}(t) s k(t) d t$, pjk < $<$, and $n_{k}$ 's are complex Gaussian with zero mean and $E\left[n_{j} n_{k}^{*}\right]=2 \sigma^{2}$ when $j=k$ and $E\left[n_{j} n_{k}^{*}\right]=2 \sigma^{2} \rho_{j k}$ when $j \neq k$. The received vector $\mathbf{y}^{(1)}$ (without hard decision) is used for MAI estimation and cancellation in the second stage.

\section{A. Conventional LPIC}

In a conventional LPIC, the interference from all other users are estimated, which are then weighed equally (with unit weight) and cancelled. The MAI estimate for the desired user $k$ in stage $m, m>1$, is obtained by multiplying $y_{j}^{\left(m-{ }^{*}\right.}$ with $\rho_{j k}$ for all $j \neq k$ and summing them up, i.e., $\sum_{j \neq k} \rho_{j k} y_{j}^{-1)}$ is the MAI estimate for the desired user $k$ in stage $m$. Accordingly, the bit decision for the fcth user after interference cancellation in the $m$ th stage is given by

$$
\hat{b}_{k}^{(m)}=\operatorname{sgn}\left(\operatorname{Re}\left(h_{k}^{*}\left(y_{k}^{(1)}-\sum_{j=1, j \neq k}^{K} \rho_{j k} y_{j}^{(m-1)}\right)\right)\right) .
$$

\section{WEIGHTED LPIC}

In a weighted LPIC, the estimate of the MAI from the $j$ th interfering user to the desired user $k$ in stage $m$ is weighted by a factor $p_{j k}^{(m)}$ before cancellation. In other words,

$\sum_{j \neq k} p_{j k}^{\langle m} \rho_{j k} y_{j}^{\prime m}$ ' is the weighted MAI estimate for the desired user $k$ in stage $m$. That is, the mth stage output of the desired user $k, y_{k}^{(m)}$, is given by

$$
y_{k}^{(m)}=y_{k}^{(1)}-\sum_{j=1, j \neq k}^{K} p_{j k}^{(\mathrm{m})} \rho_{j k} y_{j}^{(m-1)} .
$$

Note that both the conventional LPIC as well as the MF detector become special cases of the weighted LPIC forpv ${ }^{\wedge}=$ $1, \mathrm{Vj}, m$ and $p_{j k}^{(m)}=0, \mathrm{Vj}, \mathrm{m}$, respectively.

The bit decision for the desired user $k$ after weighted interference cancellation in stage $m$ is then given by

$$
\widehat{b}_{k}^{(m)}=\operatorname{sgn}\left(\operatorname{Re}\left(h_{k}^{*} y_{k}^{(m)}\right)\right) .
$$

In the following, we obtain exact expressions for the average SIRs at the output of the different stages of the weighted LPIC, which are maximized to obtain the optimum weights.

\section{A. SIR at 2 nd Stage Output}

The weighted interference cancelled output of the second stage for the desired user $k$ is given by

$$
\left.y_{k}^{(2)}=A_{k} h_{k} b_{k} \prod_{\left(1-\sum_{j=1, j \neq k}^{K}\right.} p_{j k}^{(2)} \rho_{j k}^{2}\right)+I_{2}+N_{2},
$$

where

$$
\begin{gathered}
I_{2}=\sum_{3=1, j \neq k}^{K}\left(1-p_{j k}^{(2)}\right) A_{j} h_{j} b_{j} \rho_{j k}-\prod_{j=1, j \neq k}^{K} p_{j k}^{(2)} \rho_{j k} \sum_{\substack{i=1 \\
i \neq j, k}}^{K} \rho_{i j} A_{i} h_{i} b_{i}, \\
N_{2}=n_{k}-\sum_{j=1, j \neq k}^{K} p_{j k}^{(2)} \rho_{j k} n_{j} .
\end{gathered}
$$

The terms $I_{2}$ and $N_{2}$ in (7) represent the interference and noise terms introduced due to imperfect cancellation in using the soft output values from the MF stage. Since $h$ 's are complex Gaussian, both $I_{2}$ and $N_{2}$ are linear combinations of Gaussian r.v's with zero mean. The variance of $I_{2}, \sigma_{I_{2}}^{2}$, can be obtained as

$$
\sigma_{I_{2}}^{2}=\sum_{i=1, i \neq k}^{K} A_{i}^{2}\left(\left(1-p_{i k}^{(2)}\right) \rho_{i k}-\sum_{\substack{j=1 \\ j \neq k, i}}^{K} p_{j k}^{(2)} \rho_{j k} \rho_{i j}\right)^{2},
$$

and the variance of $N_{2}, \sigma_{N_{2}}^{2}$, can be obtained as

$$
\sigma_{N_{2}}^{2}=\sigma^{2}\left(1-2 \sum_{\substack{3=1 \\ 3 \mathrm{~V}^{*}}}^{K} p_{j k}^{(2)} \rho_{j k}^{2}+\sum_{\substack{i=1 \\ i \neq k}}^{K} p_{i k}^{(2)} \rho_{i k} \sum_{\substack{3=1 \\ 3 \mathrm{~V}^{*}}}^{K} p_{j k}^{(2)} \rho_{j k} \rho_{j i}\right) .
$$

The average SIR of the desired user $k$ at the output of the second stage, $\overline{S I R}_{k}^{(?)}$, is then given by

$$
\overline{S I R}_{k}^{(2)}=\frac{A_{k}^{2}\left(1-\sum_{\substack{j=1 \\ j \neq k}}^{K} p_{j k}^{(2)} \rho_{j k}^{2}\right)^{2}}{\sigma_{I_{2}}^{2}+\sigma_{N_{2}}^{2}} .
$$

The optimum values of $p_{j k}^{(2)}, j=1,2, \cdots, K, j \neq k$ can be found by numerically maximizing the SIR expression in (12).

\section{B. SIR at 3rd Stage Output}

The soft values of the interference cancelled outputs of the different users from the 2nd stage are used to reconstruct (estimate) the MAI for the desired user $k$ in the 3rd stage, which are weighted by the factors $p_{j k}^{(3)}$ and cancelled. The 3 rd stage output of the desired user $k, y_{k}^{(2)}$, is given by

$$
y_{k}^{(3)}=A_{k} h_{k} b_{k} X+I_{3}+N_{3},
$$

where

$$
\begin{aligned}
& \mathcal{X}=1-\sum_{\substack{j=1 \\
j \neq k}}^{K} p_{j k}^{(3)} \rho_{j k}^{2}\left(1-p_{k j}^{(2)}\right)+\sum_{\substack{j=1 \\
j \neq k}}^{K} p_{j k}^{(3)} \rho_{j k} \sum_{\substack{i=1 \\
i \neq j, k}}^{K} p_{i j}^{(2)} \rho_{i j} \rho_{k i}, \\
& I_{3}=\sum_{\substack{l=1 \\
l \neq k}}^{K} \rho_{l k} A_{l} b_{l} h_{l}\left(1-p_{l k}^{(3)}\left(1-\sum_{\substack{j=1 \\
j \neq l}}^{K} p_{j l}^{(2)} \rho_{j l}^{2}\right)\right) \\
&+\sum_{\substack{l=1 \\
l \neq k}}^{K} p_{l k}^{(3)} \rho_{l k} \sum_{\substack{i=1 \\
i \neq l}}^{K} p_{i l}^{(2)} \rho_{i l} \sum_{\substack{j=1 \\
j \neq l, k, k}}^{K} \rho_{j i} A_{j} h_{j} b_{j} \\
&-\sum_{\substack{l=1 \\
l \neq k}}^{K} p_{l k}^{(3)} \rho_{l k} \sum_{\substack{j=1 \\
j \neq l, k}}^{K} \rho_{j l} A_{j} h_{j} b_{j}\left(1-p_{j l}^{(2)}\right),
\end{aligned}
$$




$$
N_{3}=n_{k}-\sum_{\substack{l=1 \\ l \neq k<}}^{K} p_{l k}^{(3)} \rho_{l k}\left(n_{l}-\sum_{\substack{3=1 \\ j \neq l}}^{K} p_{j l}^{(2)} \rho_{j l} n_{j}\right) .
$$

The terms $I_{3}$ and $N_{3}$ are linear combinations of Gaussian r.v's with zero mean and variances $\sigma_{I_{3}}^{2}$ and $\sigma_{N_{3}}^{2}$, given by

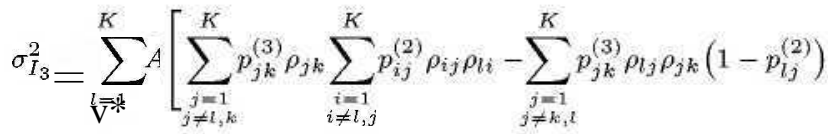

$$
\begin{aligned}
& \left.+\rho_{l k}\left(1-p_{l k}^{(3)}\left(1-\sum_{\substack{j=1 \\
j \neq l}}^{K} p_{j l}^{(2)} \rho_{j l}^{2}\right)\right)\right]^{2}, \\
& \sigma_{N_{3}}^{2}=\sigma^{2}\left(1+\sum_{l=1}^{K} \hat{p}_{l k}^{i u} p_{k l}^{2} \rho_{l k}^{2}\right)^{2} \\
& \left.+2 \sigma^{2 \prime} 1+\sum_{\substack{l=1 \\
l \neq k}}^{K} \hat{p}_{l k}^{(s)} p_{k l}^{(2)} \hat{\rho}_{l k}^{L}\right) \sum_{\substack{i=1 \\
i \neq k}}^{K} \rho_{i k}\left(-p_{i k}^{(3)} \rho_{i k}+\sum_{\substack{l=1 \\
l \neq k, i}}^{K} P l k \rho_{l k} \rho_{i l}\left(\rho_{i l}^{2)}\right)\right.
\end{aligned}
$$

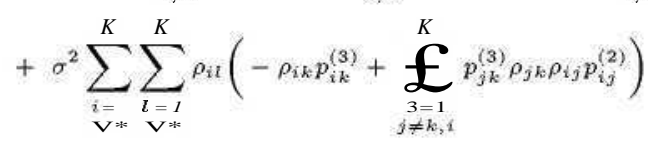

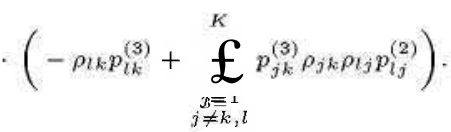

The average SIR of the desired user $k$ at the output of the third stage, $\overline{S I R}(1$, , is them by

$$
\overline{S I R}_{k}^{(3)}=\frac{A_{k}^{2} X^{2}}{\sigma_{I_{3}}^{2}+{ }_{\text {? }}} .
$$

The optimum values of $p_{j k}^{(3)}, \mathrm{j}=1,2, \cdots, K, j \neq \mathrm{fc}$, can be found by numerically maximizing the SIR expression in (19).

It is noted that the time complexity of the numerical optimization of the SIR expressions in (12) and (19) to obtain the optimum weights $p_{j k, \text { opt }}^{(2)}$ and $p_{j k \text {,opt }}^{(3)}$ is large. A less complex optimization is possible if all other users' interference is weighed equally in a given stage (yet optimally in terms of maximizing the SIR - unlike conventional LPIC which uses unit weight to all interfering users in all stages), i.e., all other users' interference in stage $m, m>1$, is weighed by the same weight $p_{k}^{(m)}$. Indeed, for this scheme we obtain the optimum weights, $p_{k, o p t}^{i_{k}}$, in closed-form, which we present in the next section. We call the $p_{j k}^{(m)}$ optimized scheme as Optimum Scheme-I and the $p_{\dot{k}}^{(m)}$ optimized scheme as Optimum Scheme-II. Since the Optimum Scheme-II weighs all users with same weight in a given stage, it is expected to loose some performance compared to the Optimum Scheme-I. However, as we will see in the Results and Discussion Section (Sec. V), the Optimum Scheme-II (for which closed-form expressions for the optimum weights are derived) performs quite close to the Optimum Scheme-I and significantly better than the conventional LPIC and the MF detector, both in near-far and as well as non-near far conditions.

\section{OPTIMUM WEIGHTS IN CLOSED-FORM}

In this section, we consider Optimum Scheme-II in which the estimates of the interference from all other users of the desired user $k$ in stage $m, m>1$, are weighed by the same weight $p_{k}^{(m)}$. Replacing $p_{j k}^{(2)}$ with $p_{k}^{(0)}$ " in (12) gives the SIR expression to optimize in the 2nd stage for Optimum SchemeII. Likewise, replacing $p_{j k}^{(3)}$ with $p_{k}^{(3)}$ in (19) gives the optimizing SIR expression for the 3rd stage.

Expressions for the optimum weights $p_{k \text { opt }}^{(2)}$ and $p_{k \text { opt }}^{(2)}$ can be obtained by differentiating (12) and (19) w.r.t. $p_{k}^{(2)}$ and $p_{k}^{(3)}$, respectively, and equating to zero. Accordingly, we obtain the expression for $p_{k}, \ldots$, , in closed-form, as

$$
p_{k, o p t}^{(2)}=\frac{c_{1}\left(1-a_{1}\right)+e_{1}}{-a_{1}\left(c_{1}+e_{1}\right)+c_{1}+d_{1}+2 e_{1}-\sigma^{2}\left(a_{1}^{2}-f_{1}\right)},
$$

where

$$
\begin{aligned}
& a_{1}=\sum_{\substack{3=1 \\
3 \mathrm{~V}^{* *}}}^{K} \rho_{j k}^{\mathbf{2}}, \quad c_{1}=\sum_{\substack{l=1 \\
\mathrm{~V} * k}}^{K} A_{l}^{2} \rho_{l k}^{\mathbf{2}}, \\
& d_{1}=\sum_{\substack{l=1 \\
l \neq k}}^{K} A_{l}^{2}\left(\sum_{\substack{j=1 \\
j \neq k, l}}^{K} \rho_{j k} \rho_{l j}\right)^{2}, \mathbf{A}=\sum_{\substack{j=1 \\
j \neq k}}^{K} \rho_{j k} \sum_{\substack{i=1 \\
\mathbf{V}^{*}}}^{K} \rho_{i j} \rho_{i k},
\end{aligned}
$$

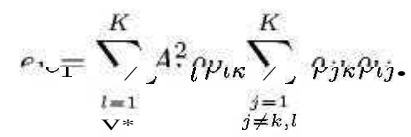

' Likewise, the closed-form expression for $p_{k}^{(3)}$ opt can be obtained as

$$
p_{k, o p t}^{(3)}=\frac{-2 a_{2} g_{2}-f_{2}-\sigma^{2}\left(2 a_{2}+t_{2}\right)}{a_{2} f_{2}+2 e_{2}+\sigma^{2}\left(a_{2} t_{2}+2 v_{2}\right)},
$$

where

$$
\begin{aligned}
& a_{2}=\sum_{\substack{3=1 \\
3 \mathrm{~V}^{*}}}^{K} \rho_{j k}^{2}\left(1-p_{j}^{(2)}\right)-\sum_{\substack{3=1 \\
3 \mathrm{~V}^{*}}}^{K} \rho_{j k} p_{j}^{(2)} \sum_{\substack{r=1 \\
r \neq j, k}}^{K} \rho_{r j} \rho_{r k}, \\
& b_{2}=1-p_{l}^{(2)} \sum_{\substack{q=1 \\
q \neq l}}^{K} \rho_{q l}^{2}, \quad g_{2}=\sum_{\substack{i=1 \\
l \neq k}}^{K} A_{l}^{2} \rho_{l k}^{2}, \\
& \mathrm{C} 2=\sum_{\substack{j=1 \\
j \neq k, l}}^{K} \rho_{j k} \rho_{l j}\left(1-p_{j}^{(2)}\right), \quad d_{2}=\sum_{\substack{j=1 \\
j \neq k, l}}^{K} \rho_{j k} p_{j}^{(2)} \sum_{\substack{r=1 \\
r \neq j, l}}^{K} \rho_{r j} \rho_{r l}, \\
& e_{2}=\sum_{\substack{l=1 \\
l \neq k}}^{K} A_{l}^{2}\left(\rho_{l k}^{2} b_{2}^{2}+\left(c_{2}-d_{2}\right)^{2}+2 \rho_{l k} b_{2}\left(c_{2}-d_{2}\right)\right) \text {, }
\end{aligned}
$$

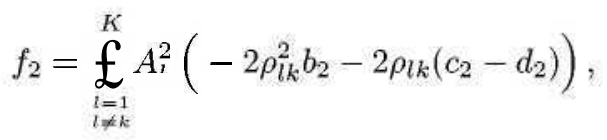

$$
\begin{aligned}
& t_{2}=2\left(u_{1}+z_{1}\right), \quad v_{2}=u_{1}^{\circ}+2 u_{1} z_{1}+t_{1}, \\
& u_{1}=\sum_{\substack{l=1 \\
\mathrm{~V} *}} p_{l}^{(2)} \mu_{l k}^{2}, \quad z_{1}=\sum_{\substack{i=1 \\
i \neq k}}^{K} \rho_{i k}\left(-\rho_{i k}+\sum_{\substack{j=1 \\
j \neq k, i}}^{K} p_{j}^{(2)} \rho_{j k} \rho_{i j}\right),
\end{aligned}
$$




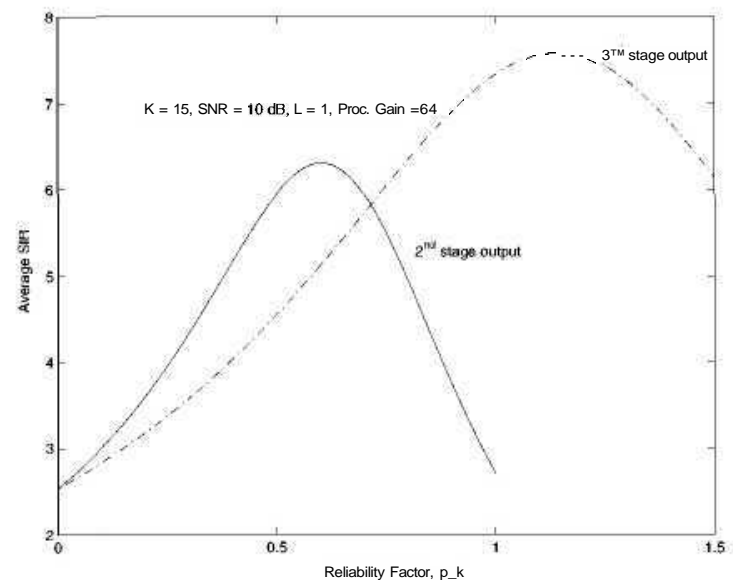

Fig. 1. Average SIR at the 2nd and 3rd stage outputs of the desired user $(k=1)$, as a function of the weight, $p_{k}^{(m)}$ Optimum Scheme-II. $K=15$. Processing gain $=64$. Random spreading sequences. SNR $=10 \mathrm{~dB}$.

$t_{1}=\sum_{\substack{i=1 \\ i \neq k}}^{K} \sum_{\substack{l=1 \\ l \neq k}}^{K} \rho_{i l}\left(-\rho_{i k}+\sum_{\substack{j=1 \\ j \neq k_{i}}}^{K} p_{j}^{(2)} \rho_{j k} \rho_{j}\right)\left(-\rho_{l k}+\sum_{\substack{j=1 \\ j \neq k, l}}^{K} p_{j}^{(2)} \rho_{j k} \rho_{l j}\right)$.

In Fig. 1, using Eqns. (12) and (19),we plot the average SIR at the 2 nd $(m=2)$ and 3rd ( $m=3)$ stage outputs of the Optimum Scheme-II for the desired user $(k=1)$, as a function of the weight $p_{\dot{k}}^{(m)}$ for the case of $K=15$ users using random spreading sequences of processing gain 64 and $\mathrm{SNR}=A^{2} / \sigma^{2}$ $=10 \mathrm{~dB}$. It is noted that the maximum interference cancelled output SIR increases as the number of stages increases (i.e., $\overline{S I R}_{\text {kopt }}^{(\prime)}>S I R_{\text {kopt }}^{(2)}$, which is intuitively expected).

\section{A. Probability of Bit Error}

The probability of bit error of the desired user $k$ at the output of the $m$ th stage can be obtained in terms of the optimized SIR as

$$
\left.\mu_{k}^{(m)}=\mathbf{i}_{2}^{\cdot} / 1-\sqrt{\frac{\overline{S I R}_{k, o p t}^{(m)}}{1+\overline{S I R}_{k, o p t}^{(m)}}}\right) .
$$

where $\overline{S I R}_{\text {kopt }}^{(m)}$ is the output SIR when the optimum weight $p_{k, \text { opt }}^{(m)}$ is used.

Suppose we consider receive diversity with $L$ equal-energy i.i.d paths. In this case, cancellation is done on each path and the resulting outputs are coherently combined. Accordingly, the bit decision for the desired user $k$ in stage $m$ is given by

$$
\hat{b}_{k}^{(m)}=\operatorname{sgn}\left(\operatorname{Re}\left(\sum_{=1}^{L} h_{k}^{l}{ }^{*} y_{k}^{(m)_{l}}\right)\right),
$$

where $h_{k}^{l}$ denotes the fcth user's complex channel coefficient on the $l$ th receive antenna path, and $y_{k}^{(m)}$ denotes the fcth user's interference cancelled output of the mth stage on the $\mathrm{fth}$ receive antenna path, given by

$$
y_{k}^{(m)_{l}}=y_{k}^{(1)_{l}}-\sum_{j=1, j \neq k}^{K} p_{j k}^{(\mathrm{m}) ;} \rho_{j k} y_{j}^{(m-1)_{l}} .
$$

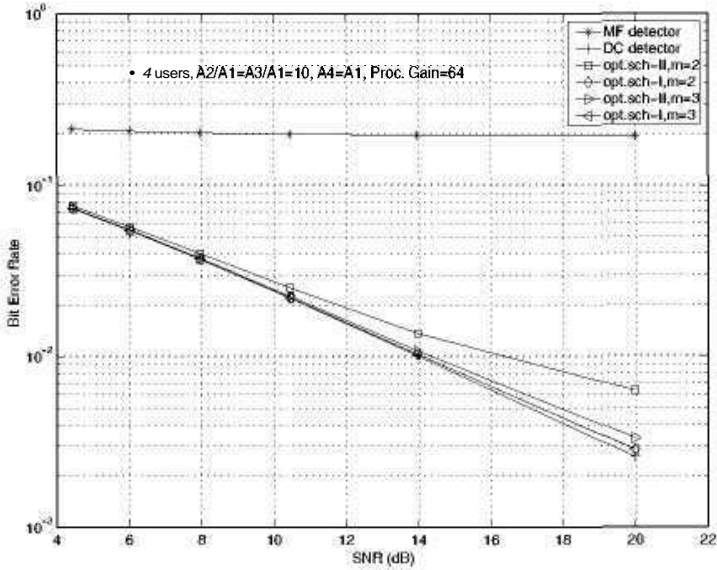

Fig. 2. BER performance of SIR-optimized WLPIC schemes. Optimum Schemes I and II. $L=1, K=4$, Proc.Gain $=64$. Near-far effect: $A_{2} / A_{1}=A_{3} / A_{1}=10, A_{4}=A 1$.

On each receive antenna path, the expressions for the variances due to interference, $\sigma_{I_{m}}^{2}$, and due to noise, $\sigma_{N_{m}}^{2}$, remain the same as those for the flat fading case given before. The probability of bit error for the desired user $k$ with $L$ equalenergy diversity paths can be obtained as (similar to obtaining Eq. 3.140 in [1])

$P_{k}^{(m)}=\frac{1}{2}-\frac{1}{2} \sqrt{\frac{\overline{\operatorname{SIR}}_{k, o p t}^{(m)}}{1+\overline{\operatorname{SIR}}_{k, o p t}^{(m)}}}\left(1+\sum_{n=1}^{L-1} \frac{1 \cdot 3 \cdot 5 \cdots(2 n-1)}{n ! 2^{n}\left(1+\overline{\operatorname{SIR}}_{k, o p t}^{(m)}\right)^{n}}\right) \cdot(25)$

\section{$V$. RESULTS AND DISCUSSIONS}

In this section, we present the BER performance of the SIRoptimized weighted LPIC (WLPIC) schemes presented in the previous sections. In Fig. 2, we plot the BER of the Optimum Scheme-I ( $p_{j k}^{(m)}$ optimized scheme) and Optimum SchemeII ( $p_{k}$ optimized scheme) as a function of SNR on flat Rayleigh fading $(L=1)$, for the 2 nd $(m=2)$ and $3 \mathrm{rd}$ $(m=3)$ stages. The number of users is $K=4$ with near-far effect such that users 2 and 3 have 10 times more amplitude than users 1 and 4 (i.e., $A_{2} / A_{1}=A_{3} / A_{1}=10, A_{4}=A_{1}$ ). In all the performance plots, user 1 is taken to be the desired user. All the BER plots are based on the analytical expressions in (22) using the appropriate optimized SIR values. In all the numerical results presented here, we assign different random spreading sequences of processing gain 64 to different users, and the cross-correlation coefficients are computed for these random sequences. We have obtained the BER through simulations as well (we found close match between analysis and simulation results which is expected since the expressions are exact and there is no approximation involved). The performance of the MF detector as well as the decorrelating (DC) detector are also shown for comparison.

From Fig. 2, it is observed that the 2nd stage of the Optimum Scheme-I itself performs very close to that of the DC detector. In fact, since the number of users is small, the Optimum Scheme-I is able to remove most interference in the 2nd stage itself and the 3rd stage provides very little (almost 


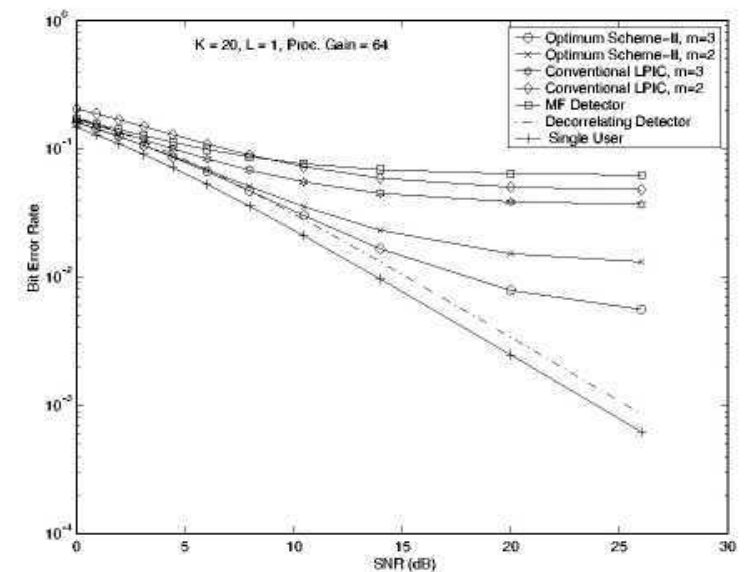

Fig. 3. Comparison of the BER performance of SIR-optimized WLPIC Optimum Scheme-II as a function of SNR with that of MF detector and conventional LPIC. $K=20, L=1$. No near-far effect: $A_{1}=A_{2}=\cdots \cdot=A_{K}$.

nil) improvement. It must however be pointed out that the time complexity of the optimization in Optimum Scheme-I gets increasingly large for large $K$. The Optimum SchemeII, on the other hand, performs little worse than Optimum Scheme-I as expected. In fact, the 3rd stage performance of Optimum Scheme-II is quite close to the 2nd stage performance of Optimum Scheme-I as well as the DC detector performance. So the cancellation using Optimum Scheme-II is found to be quite effective while having the advantage of obtaining the optimum weights in closed-form.

In Fig. 3, we illustrate a BER performance comparison of the Optimum Scheme-II as a function of SNR with the MF detector as well as the conventional LPIC on flat Rayleigh fading $(L=1)$ for $K=20$ users without near-far effect (i.e., $\left.A_{1}=A_{2}=\cdots=A K\right)$. The performance of the MF and DC detectors as well as the single user performance are also plotted for comparison. From Fig. 3, the following observations can be made. At high SNRs, the 2nd stage of the conventional LPIC performs better than the MF detector, due to accurate MAI estimates in good channel conditions (i.e., high SNRs). However, at low SNRs (SNRs $<9 \mathrm{~dB}$ in Fig. 3), the 2nd stage of the conventional LPIC performs poorer than the MF detector, which happens due to poor accuracy of the MAI estimates in the early stages of the PIC, particularly when channel conditions are poor (i.e., at low SNRs). This performance behavior improves in the 3rd stage of the conventional LPIC which performs better than the MF detector for all SNRs $>0$ $\mathrm{dB}$. It is however noted that the SIR-optimized WLPIC Optimum Scheme-II clearly outperforms both the MF detector as well as the conventional LPIC. In fact, even the 2nd stage of the Optimum Scheme-II outperforms the 3rd stage of the conventional LPIC, and the performance of the 3rd stage of the Optimum Scheme-II tends closer to that of the DC detector.

Fig. 4 illustrates the BER performance comparison of the Optimum Scheme-II as a function of the number of users $K$ with the MF detector as well as the conventional LPIC, for $L=2$ at an SNR of $10 \mathrm{~dB}$ in a near-far scenario where users 2 and 4 transmit with 4 times more amplitude than the other users (i.e., $A_{2} / A_{1}=A_{4} / A_{1}=4, A_{1}=A_{3}=A_{5}=\bullet \bullet=A_{K}$ ).

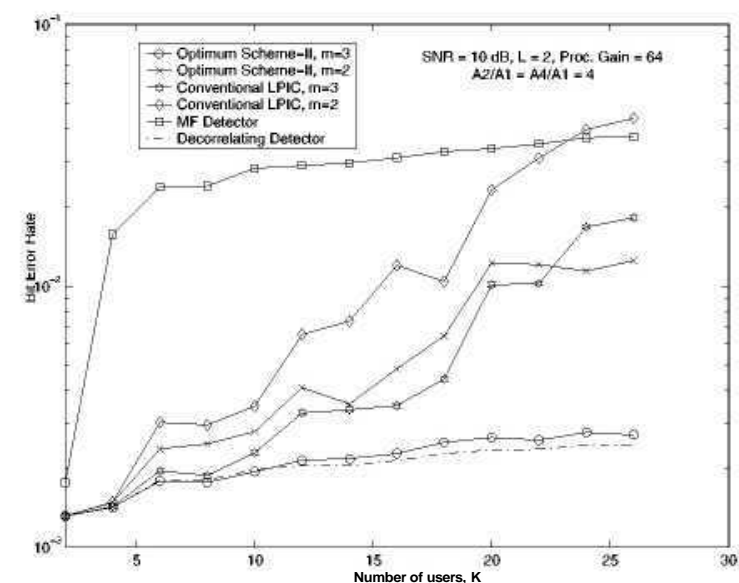

Fig. 4. Comparison of the BER performance of SIR-optimized WLPIC Optimum Scheme-II as a function of number of users with that of MF detector and conventional LPIC. $L=2$, SNR $=10 \mathrm{~dB}$. Near-far effect: $A_{2} / A_{1}=A_{4} / A_{1}=4, A_{1}=A_{3}=A_{5}=\cdots=A_{K}$.

From Fig. 4, we observe that the Optimum Scheme-II clearly performs better than the MF detector and conventional LPIC, with its 3rd stage performing quite close to the DC detector's performance.

\section{CONCLUSIONS}

We presented SIR-optimized weighted linear parallel interference cancellation (LPIC) schemes where the estimated interference from each user is weighted by a factor before cancellation on Rayleigh fading and diversity channels. We obtained exact expressions for the SIR at the output of the cancellation stages which we maximized to obtain the optimum weights for each interfering user in each cancellation stage. For a system that uses same weight for all interfering users in a given stage, we obtained exact closed-form expressions for the optimum weights for the different stages of the LPIC. We showed that this SIR-optimized weighted LPIC scheme clearly outperforms the MF detector as well as the conventional LPIC scheme, in both near-far as well as non-near-far conditions on Rayleigh fading and diversity channels.

\section{REFERENCES}

[1] S. Verdu, Multiuser Detection, Cambridge University Press, 1998.

[2] M. Varanasi and B. Aazhang, "Multistage detection in asynchronous code-division multiple-access," IEEE Trans. Commun., vol. 38, pp. 509-519, April 1990

[3] D. R. Brown, M. Motani, V. Veeravalli, H. V. Poor, and C. R. Johnson, Jr., "On the performance of linear parallel interference cancellation," IEEE Trans. Inf. Theory, vol. 47, no. 5, pp. 1957-1970, July 2001.

[4] D. Divsalar, M. K. Simon, and D. Raphaeli, "Improved parallel interference cancellation for CDMA," IEEE Trans. Commun., vol. 46, no. 2, pp. 258-268, February 1998.

[5] P. G. Renucci and B. D. Woerner, "Optimization of soft interference cancellation for DS-CDMA," Elect. Letters, vol. 34, no. 8, pp. 731733, April 1998.

[6] D. Guo, L. K. Rasmussen, S. Sun, T. J. Lim, and C. Cheah, "MMSEbased linear parallel interference cancellation in CDMA," Proc. IEEE ISSSTA, pp. 917-921, September 1998.

[7] Y-H. Li, M. Chen, and S-X. Chen, "Determination of cancellation factors for soft decision partial PIC detector in DS-CDMA systems," Electronics Letters, pp. 239-241, 3 February 2000.

[8] M. Ghotbi and M. R. Soleymani, "A simple method for computing partial cancellation factors in CDMA using PPIC receiver," Proc. IEEE ICASSP, pp. 973-976, 2004. 